\title{
Beyond allostery: Catalytic regulation of a deoxyribozyme through an entropy-driven DNA amplifier
}

\author{
Grace Eckhoff', Vlad Codrea ${ }^{1}$, Andrew D Ellington ${ }^{1,2^{*}}$, Xi Chen $^{2^{*}}$
}

\begin{abstract}
The programmability and replicability of RNA and DNA have respectively enabled the design and selection of a number of allosteric ribozymes and deoxyribozymes. These catalysts have been adapted to function as signal transducers in biosensors and biochemical reaction networks both in vitro and in vivo. However, allosteric control of nucleic acid catalysts is currently limited by the fact that one molecule of effector (input) generally regulates at most one molecule of ribozyme or deoxyribozyme (output). In consequence, allosteric control is usually inefficient when the concentration of input molecules is low. In contrast, catalytic regulation of protein enzymes, as in protein phosphorylation cascades, generally allows one input molecule (e.g., one kinase molecule) to regulate multiple output molecules (e.g., kinase substrates). Achieving such catalytic signal amplification would also be of great utility for nucleic acid circuits. Here we show that allosteric regulation of nucleic acid enzymes can be coupled to signal amplification in an entropy-driven DNA circuit. In this circuit, kinetically trapped DNA logic gates are triggered by a specific sequence, and upon execution generate a peroxidase deoxyribozyme that converts a colorless substrate (ABTS) into a green product $\left(\mathrm{ABTS}^{++}\right)$. This scheme provides a new paradigm for the design of enzyme-free biosensors for point-of-care diagnostics.
\end{abstract}

\section{Findings}

A variety of functional nucleic acids have been engineered over the past two decades, including not only simple binding elements (aptamers [1,2]) and catalysts (ribozymes [3] and deoxyribozymes [4]), but also more 'intelligent' molecular parts, such as aptamer beacons and allosteric ribozymes that can sense biomolecules $[5,6]$, process molecular information $[7,8]$, and regulate biochemical systems [9]. However, most regulatory nucleic acid elements are based on allosteric control, which has a fundamental limitation: one input molecule generally yields only one output molecule. Such stoichiometric or sub-stoichiometric regulation is often insufficient for effective metabolic regulation or diagnostic signal transduction, especially when the concentrations of input molecules are low.

\footnotetext{
* Correspondence: andy.ellington@mail.utexas.edu; xichen@mail.utexas.edu 'Institute for Cellular and Molecular Biology, University of Texas at Austin, Austin, Texas 78712, USA

2Department of Chemistry and Biochemistry, University of Texas at Austin, Austin, Texas 78712, USA

Full list of author information is available at the end of the article
}

In contrast, natural catalytic cascades, such as the phosphorylation of proteins by kinases, readily amplify low input signals. Although in principle ribozymes and deoxyribozymes could participate in similar cascades as catalysts [10-12], no generalizable method for implementing such cascades has yet been established. On the other hand, DNA and RNA can catalyze chemical reactions not only by forming intricate tertiary structures, but also by simply forming Watson-Crick base pairs. In fact, by serving as a hybridization template, DNA can control and catalyze a wide range of chemical reactions [13], some of which can yield products capable of regulating downstream reactions. More recently, Zhang and coworkers have designed a scheme for highly efficient, enzyme-free, entropy-driven catalytic reactions that relies only on the dynamic hybridization of DNA strands [14-17]. Because of its chemical simplicity, this scheme is expected to allow the development of enzyme-free DNA circuits substantially more complex and robust [18] than previous enzyme-dependent examples [19-22]. Similar strand displacement-based schemes using DNA hairpins as substrates have also been devised $[23,24]$. 
Moreover, it has been shown that the principles used to construct aptamer beacons and allosteric ribozymes [5] can also be applied to these schemes in order to create DNA circuits that use molecules other than nucleic acids as input signals [23].

While DNA circuits may prove useful as diagnostics, strand-exchange reactions have so far been monitored by sophisticated analytical devices. As an example of the possibilities available for signal transduction, we convert one molecule of input DNA to multiple molecules of a peroxidase deoxyribozyme $[12,25]$ that in turn converts the colorless substrate 2,2'-azino-bis(3-ethylbenzthiazoline-6-sulphonic acid) (ABTS) into the readily observed green product $\mathrm{ABTS}^{*+}$. We also demonstrate that an entropy-driven catalytic DNA circuit can function as a generic signal amplification module that allows lower concentrations of input molecules to control the production of higher concentrations of deoxyribozymes, and thus exceed the limit of stoichiometric allosteric control. The system is qualitatively sensitive to mismatches in the input nucleic acid, suggesting that the combined entropy-driven strand exchange and allosteric deoxyribozyme circuit might facilitate the future development of enzyme-free, point-of-care diagnostic assay system [26-28].

As a starting point for the development of a joint entropy-driven strand exchange and allosteric deoxyribozyme circuit, we began with the one-step, entropy- driven DNA catalytic circuit shown in Figure 1A, similar to that developed by David Zhang and Erik Winfree [16]. The functional segments of each DNA strand are called 'domains' and are designated by numbers. Each domain is further divided into two sub-domains: a 6-ntlong toehold $[29,30]$ sub-domain $(t)$, and a 14 15-ntlong branch migration sub-domain $(b)$. The sequences for all sub-domains are summarized in Table 1.

In this circuit, the amplification module contains a Gate duplex at 100 to $200 \mathrm{nM}$, and a Fuel strand at a higher concentration ( $300 \mathrm{nM}$ to $1 \mu \mathrm{M}$ ); this will allow the entropy-driven execution of the circuit. The Gate duplex in turn consists of a Dock strand and an Invader strand. Although in principle the Fuel can displace the Invader from the Gate, this reaction is very slow due to the stability of DNA duplex. However, in the presence of even low concentrations of an input (the Trigger strand), the sub-domain $1 t$ at the 3 ' end of the Trigger can bind the toehold sub-domain $1 t^{*}$ at the $5^{\prime}$ end of the Dock, and initiate a branch migration reaction in which the Trigger displaces the Invader from the Dock. After the Invader dissociates, a Fuel strand can interact with the Dock via the sub-domain $2 t$ at its 5 ' end, and initiate a backward branch migration to displace, and thus recycle, the Trigger (with the concomitant formation of the Waste_1 duplex). The recycled Trigger can then catalyze another cycle of exchange between the Invader and the Fuel on the Dock. Thus,

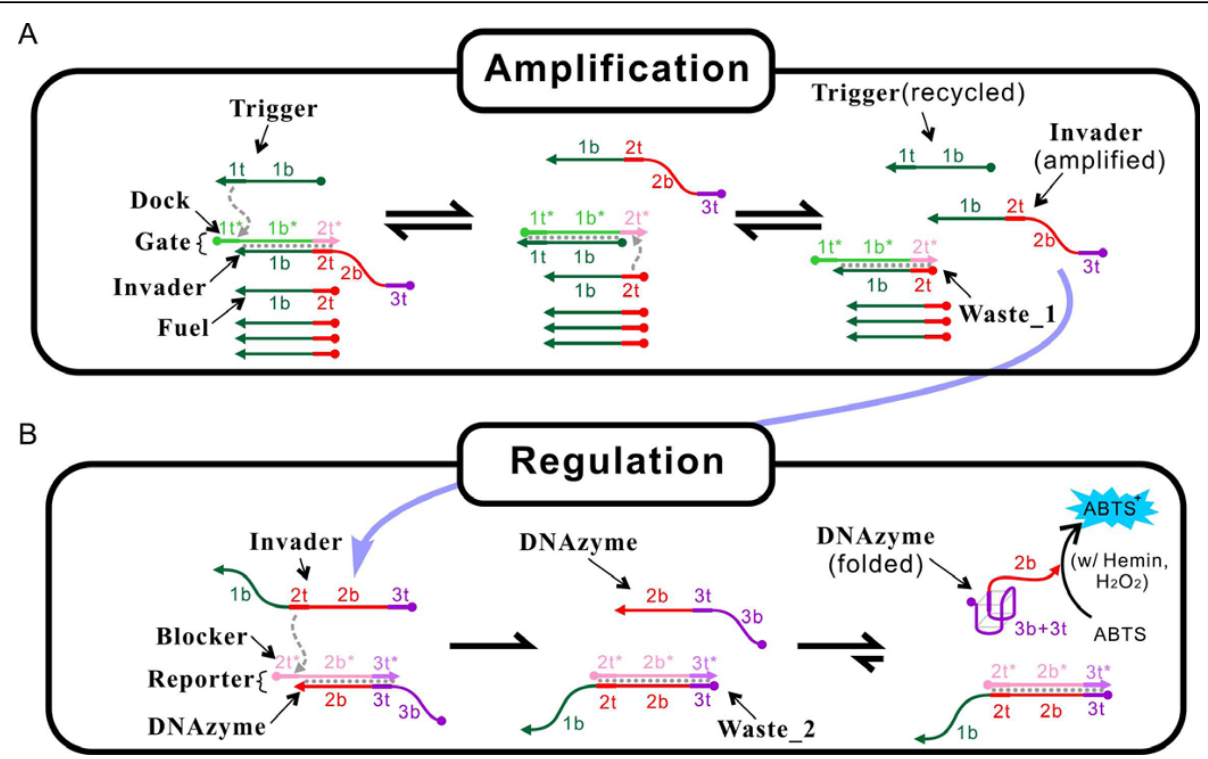

Figure 1 Entropy-driven deoxyribozyme circuit. (A) Scheme for the amplification module, based on an entropy-driven, strand-exchange amplifier. The functional domains within each DNA strand have been color-coded (see Table 1 for the sequence of each domain).

Complementary domains are represented by light and dark variation of the same color (i.e., light green pairs with dark green). An asterisk in the names of sub-domains indicates complementarity (i.e., sub-domain $1 t^{*}$ is complementary to sub-domain $1 t$ ). Gray dashed arrows indicate where toehold binding should initiate. Arrows and dots on DNA strands represent 3' termini and 5' termini, respectively. (B) Scheme for the reporter module based on an allosteric G-quadruplex peroxidase. The domain containing the G-quadruplex is colored in purple. 
Table 1 Sequence of DNA sub-domains used in this work

\begin{tabular}{cc}
\hline Sub-domain & Sequence \\
\hline $1 \mathrm{t}$ & TCTCCA \\
\hline $1 \mathrm{~b}$ & ATTCAATACCCTACG \\
\hline $2 \mathrm{t}$ & CGTCTC \\
\hline $2 \mathrm{~b}$ & CGTAAATATTGGCG \\
\hline $3 \mathrm{t}^{\dagger}$ & GAGGGA \\
\hline $3 \mathrm{~b}$ & CTGGGAGGGAGG \\
\hline
\end{tabular}

${ }^{+}$The domain 3t of fDNAzyme is AGGGA instead of GAGGGA, in order to avoid quenching of the FAM by the adjacent $5^{\prime} \mathrm{G}$.

the net reaction of these two processes is the reversible reaction Gate + Fuel $=$ Invader + Waste 1 1, where the Trigger serves as a catalyst to accelerate this otherwise very slow reaction, and thereby reach an equilibrium where a substantial amount of Invader is released.

The product of the amplification module, namely the Invader strand, can then enter the allosteric deoxyribozyme module (Figure 1B) which consists solely of a Reporter duplex. The reporter duplex is formed by the hybridization of the DNAzyme strand and the Blocker strand. The DNAzyme strand has a catalytic domain 3 (colored dark purple in Figure 1B) that can fold into a Gquadruplex and exhibit peroxidase activity in the presence of hemin. However, the activity of the deoxyribozyme is inhibited by hybridization to the Blocker strand in the Reporter duplex. The Invader strand produced by the amplification module can displace the DNAzyme from the Reporter duplex with the concomitant formation of the Waste_2 duplex. Only upon Invadermediated displacement can the DNAzyme strand undergo a conformational change and fold into an active G-quadruplex conformation and (in the presence of hemin and $\mathrm{H}_{2} \mathrm{O}_{2}$ ) oxidize the colorless substrate ABTS to produce the readily observed green product $\mathrm{ABTS}^{\bullet+}$. Thus, the overall function of the combined circuit is to amplify one molecule of Trigger into multiple molecules of G-quadruplex deoxyribozyme and colored product.

In order to assay the functionality of the amplification module we monitored the formation of Invader in realtime. The fReporter duplex (Figure 2A) was synthesized with a FAM-labeled fDNAzyme strand and a quencherlabeled qBlocker strand (Figure 2A). Upon addition of Invader the fDNAzyme should be displaced from the qBlocker, resulting in a stoichiometric (not catalytic) increase in fluorescent signal. Although $100 \mathrm{nM}$ of fReporter showed some fluorescent background (defined as $1 \mathrm{RFU})$ due to incomplete quenching, the addition of Invader increased the fluorescence signal up to $2.5 \mathrm{RFU}$ at saturating concentrations $(\geq 100 \mathrm{nM}$, data not shown). In contrast, the addition of $100 \mathrm{nM}$ Gate (with or without $1 \mu \mathrm{M}$ Fuel; Figure $2 \mathrm{~B}$, blue trace and green trace, respectively) to the fReporter caused only a small increase in fluorescent signal (a process commonly called 'circuit leakage,' see Section 2 of [Additional file $1]$ ), likely due to the presence of small amounts of free Invader, to the displacement of fDNAzyme by the Gate duplex in a toehold-independent manner, and/or to the uncatalyzed exchange between the Invader and the Fuel. However, addition of 5 to $20 \mathrm{nM}$ of Trigger in the presence of Fuel substantially accelerated the release of Invader in a dose-dependent manner, as shown by the much faster increase in fluorescent signal (Figure $2 \mathrm{~B}$, red traces). To confirm that the Trigger was in fact being recycled to achieve amplification, we carried out the same reaction in the absence of Fuel (Figure 2C). As expected, although there were initial bursts of fluorescence signal due to the stoichiometric or sub-stoichiometric displacement of Invader by the Trigger, the signal did not increase steadily (compare red and magenta traces in Figure 2C).

Figure 2D directly compares the extent of reaction between different samples after $1 \mathrm{hr}$. Notably, the increase of fluorescent signal by $5 \mathrm{nM}$ Trigger in the presence of Fuel was $~ 0.3$ RFU (comparing samples \#3 and \#4), which corresponded to the production of $\sim 20 \mathrm{nM}$ of Invader. Thus, in the context of the circuit as a whole 5 nM Trigger presumably produced $20 \mathrm{nM}$ Invader, and thus the original signal was amplified by $(20 \mathrm{nM} / 5 \mathrm{nM}=)$ 4-fold within an hour. While this value is relatively small compared to other methods, it should be noted that the extent of amplification was primarily limited by the high, uncatalyzed background reaction which in $1 \mathrm{hr}$ produced a signal equivalent to that produced by $\sim 25 \mathrm{nM}$ Invader. This background can be tuned by increasing the purity of the Gate duplex (see Section 2 of [Additional file 1]), and by 'clamping' and 'padding' the duplex to decrease non-specific strand exchange [24].

More important to the function of this chemical system than amplification alone was the ability to modularly generate an optical signal, and the ability to program mismatch discrimination for the Trigger input. The allosteric deoxyribozyme module was adapted to the whole circuit shown in Figure 1 by mixing all of the DNA species together in $10 \mu \mathrm{L}$ volume at $10 \times$ final concentration for $30 \mathrm{~min}$, then adding $10 \mu \mathrm{L}$ of $2 \mu \mathrm{M}$ hemin followed by $20 \mathrm{~min}$ incubation at room temperature ('DNA/hemin mixture'). These sequential incubations should have allowed the formation of deoxyribozymehemin complexes from any DNAzyme strands freed due to the execution of the circuit. To initiate the detection reaction, $80 \mu \mathrm{L}$ substrate solution containing $\mathrm{H}_{2} \mathrm{O}_{2}$ and ABTS was added to the DNA/hemin mixture, making a final volume of $100 \mu \mathrm{L}$. The final concentrations of hemin, $\mathrm{H}_{2} \mathrm{O}_{2}$, and ABTS were $200 \mathrm{nM}, 2 \mathrm{mM}$ and $2 \mathrm{mM}$, respectively, and a colorimetric signal could be easily read within 15 minutes. To ensure that signal could be 


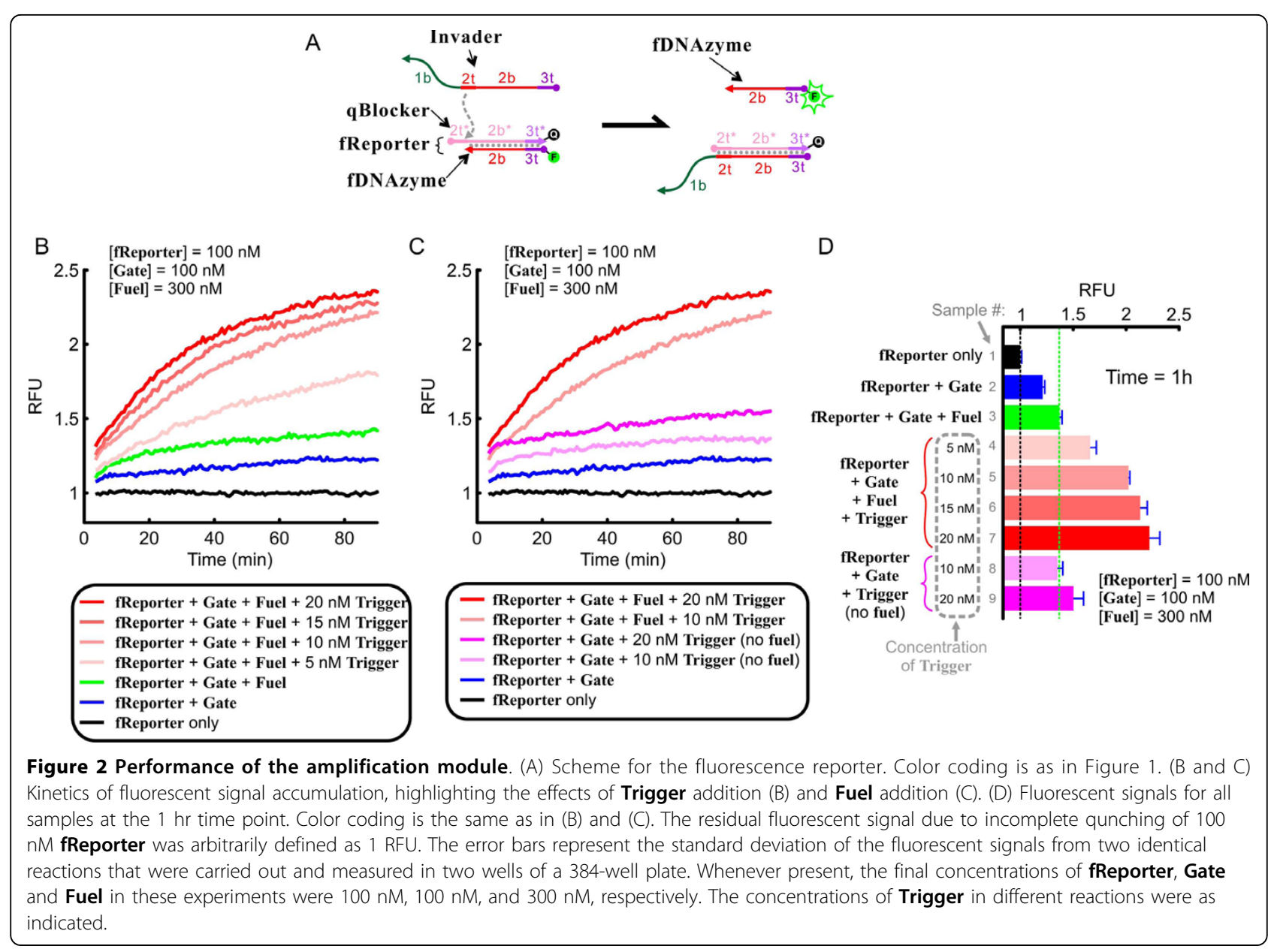

easily detected by eye, $200 \mathrm{nM}$ of DNAzyme strand was shown to exhibit strong peroxidase activity in the presence of $200 \mathrm{nM}$ hemin (Figure 3, sample \#5), while 200 $\mathrm{nM}$ of Reporter duplex was inactive under the same conditions (Figure 3, sample \#1).

The combined chemical system could thus be assayed for the ability to detect input Trigger strands. Notably, in the presence of Gate and Fuel, as little as $25 \mathrm{nM}$ of Trigger was able to produce sufficient peroxidase activity for visual observation of $\mathrm{ABTS}^{\bullet+}$ formation after only 15 min of reaction (Figure 3, sample \#6). Observed peroxidase activity further increased when the Trigger concentration was increased to $50 \mathrm{nM}$ (Figure 3, samples \#7 9). The circuit could be shown to be dependent upon both Trigger and Fuel. In the absence of Fuel, 50 $\mathrm{nM}$ of Trigger alone did not yield sufficient peroxidase activity for visual observation (Figure 3, sample \#13). In the absence of Trigger, $200 \mathrm{nM}$ of Gate only restored a small fraction of peroxidase activity even with the presence of $500 \mathrm{nM}$ of Fuel (Figure 3, sample \#2 and \#3). As a positive control, the addition of $200 \mathrm{nM}$ of Invader to $200 \mathrm{nM}$ of Gate and $200 \mathrm{nM}$ of Reporter almost completely restored peroxidase activity (Figure 3, compare sample \#4 with sample \#5).

Zhang and Winfree have shown that single-nucleotide mutations, insertions, and deletions in the catalyst strand can result in 10 - to 300 -fold reductions in its efficiency in catalyzing strand exchange [17]. These reductions in signal observed with single-nucleotide mismatches are comparable to other well-developed DNA sensors [26-28,31-33]. Consistent with these findings, we observed that when a single nucleotide was changed in the toehold region (Figure 3 , inset), the mutant Trigger did not yield any peroxidase activity above background even at $150 \mathrm{nM}$ concentration (Figure 3, sample \#10 12).

In summary, by coupling an entropy-driven DNA circuit with deoxyribozyme catalysis we created a catalytic cascade akin to phosphorylation-based protein regulation in biology. Optimization of circuit design and purification methods (see Section 2 of [Additional file 1]) coupled with cascaded and autocatalytic amplification schemes $[14,24]$ should greatly improve circuit performance. Overall, these results suggest that entropy-driven 


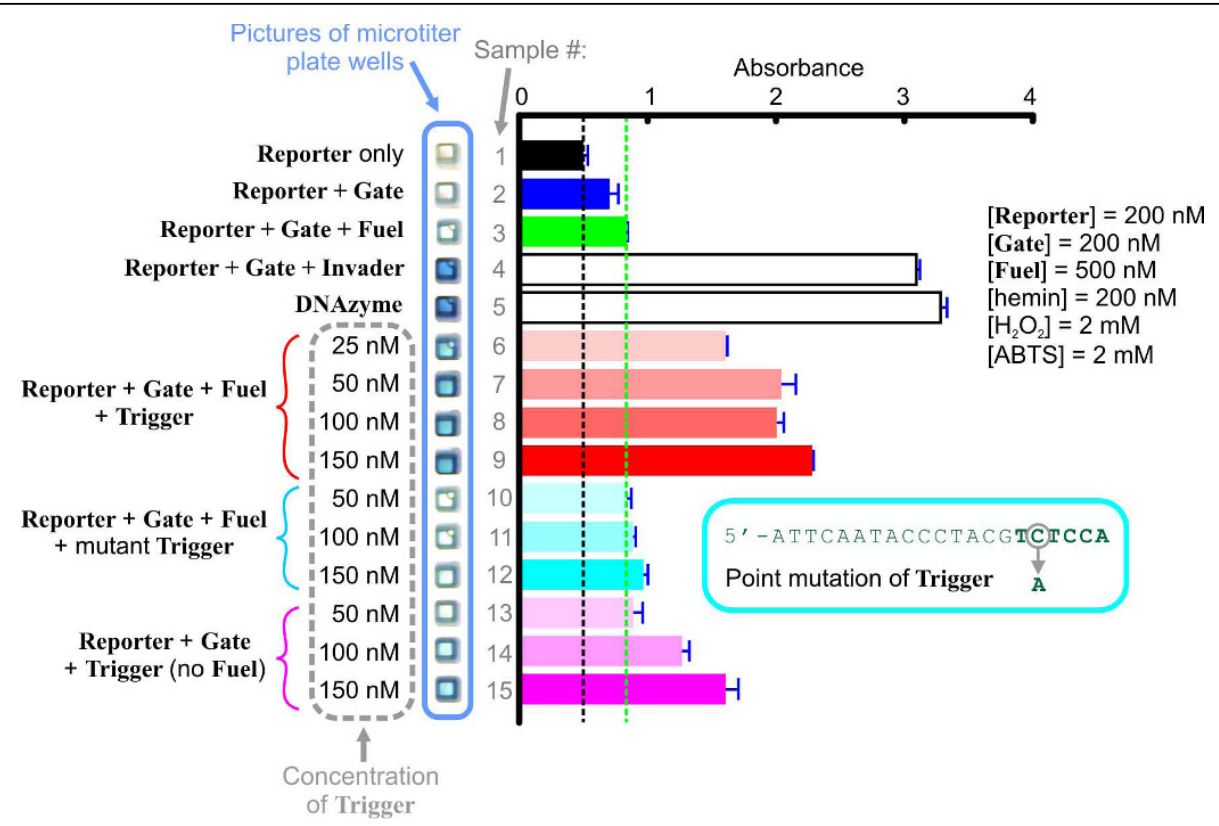

Figure 3 Performance of the entropy-driven deoxyribozyme circuit. Sample numbers (as indicated in the text) are listed next to the bars. Color coding is as in Figure 2D. The pictures of the microtiter-plate wells containing different samples are shown in the blue box. The absorbance readings (at $414 \mathrm{~nm}$ ) shown in the bar graph were taken 10 min after the $\mathrm{H}_{2} \mathrm{O}_{2}$ and ABTS were added to the DNA/hemin mixture and the pictures were taken $15 \mathrm{~min}$ after the $\mathrm{H}_{2} \mathrm{O}_{2}$ and $\mathrm{ABTS}$ were added to the DNA/hemin mixture. The inset shows the point mutation in the mutant Trigger. The toehold sequence is highlighted in bold. Whenever present, the final concentrations of Reporter, Gate, Fuel, Invader, and DNAzyme in these experiments were 200 nM, 200 nM, 500 nM, 200 nM, and 200 nM, respectively. The concentrations of Trigger and mutant Trigger in different reactions were as indicated.

DNA circuits coupled with colorimetric readouts could lead to new point-of-care nucleic acid detection methods, including field-based qualitative plus/minus discrimination between mutant and wild-type alleles.

\section{Additional material}

Additional file 1: Supplementary Text. This document provides detailed experimental procedures and discussions on purification methods and circuit leakage.

\section{Acknowledgements}

We thank Dr. David Yu Zhang for helpful discussions on the design of the amplification module, and Dr. Bingling Li for her suggestions on the optimization of conditions for the colorimetric assay. This work was supported by the Welch foundation (F-1654) and National Institute of Health (GM077040-08, EB007689). XC was partly supported by the Graduate School Continuing Fellowships of the University of Texas at Austin.

\section{Author details}

${ }^{1}$ Institute for Cellular and Molecular Biology, University of Texas at Austin, Austin, Texas 78712, USA. ${ }^{2}$ Department of Chemistry and Biochemistry, University of Texas at Austin, Austin, Texas 78712, USA.

\section{Authors' contributions}

XC proposed the study, carried out preliminary experiments and drafted the manuscript. GE carried out all the experiments shown in the manuscript. VC designed the sequence of DNA strands. ADE suggested experiments, managed the research enterprise, and critically revised the manuscript.

\section{Competing interests}

The authors declare that they have no competing interests.

Received: 10 June 2010 Accepted: 1 October 2010

Published: 1 October 2010

\section{References}

1. Ellington AD, Szostak JW: In vitro selection of RNA molecules that bind specific ligands. Nature 1990, 346:818-822.

2. Tuerk C, Gold L: Systematic evolution of ligands by exponential enrichment: RNA ligands to bacteriophage T4 DNA polymerase. Science 1990, 249:505-510.

3. Chen X, Li N, Ellington AD: Ribozyme catalysis of metabolism in the RNA world. Chem Biodivers 2007, 4:633-655.

4. Breaker RR, Joyce GF: A DNA enzyme that cleaves RNA. Chem Biol 1994, 1:223-229.

5. Liu J, Cao Z, Lu Y: Functional nucleic acid sensors. Chem Rev 2009, 109:1948-1998.

6. Willner I, Zayats M: Electronic aptamer-based sensors. Angew Chem Int Ed Engl 2007, 46:6408-6418.

7. Stojanovic MN, Stefanovic D: A deoxyribozyme-based molecular automaton. Nat Biotechnol 2003, 21:1069-1074.

8. Mao C, LaBean TH, Relf JH, Seeman NC: Logical computation using algorithmic self-assembly of DNA triple-crossover molecules. Nature 2000, 407:493-496.

9. Isaacs FJ, Dwyer DJ, Collins JJ: RNA synthetic biology. Nat Biotechnol 2006, 24:545-554.

10. Levy M, Ellington AD: Exponential growth by cross-catalytic cleavage of deoxyribozymogens. Proc Natl Acad Sci USA 2003, 100:6416-6421.

11. Lam BJ, Joyce GF: Autocatalytic aptazymes enable ligand-dependent exponential amplification of RNA. Nat Biotechnol 2009, 27:288-292.

12. Teller C, Shimron S, Willner I: Aptamer-DNAzyme hairpins for amplified biosensing. Anal Chem 2009, 81:9114-9119. 
13. Grossmann TN, Strohbach A, Seitz O: Achieving turnover in DNAtemplated reactions. Chembiochem 2008, 9:2185-2192.

14. Zhang DY, Turberfield AJ, Yurke B, Winfree E: Engineering entropy-driven reactions and networks catalyzed by DNA. Science 2007, 318:1121-1125.

15. Zhang DY, Winfree E: Dynamic allosteric control of noncovalent DNA catalysis reactions. J Am Chem Soc 2008, 130:13921-13926.

16. Zhang DY, Winfree E: Control of DNA strand displacement kinetics using toehold exchange. J Am Chem Soc 2009, 131:17303-17314.

17. Zhang DY, Winfree E: Robustness and modularity properties of a noncovalent DNA catalytic reaction. Nucleic Acids Res 2010, 38:4182-4197.

18. Qian L, Winfree E: A Simple DNA Gate Motif for Synthesizing Large-Scale Circuits. Lect Notes Comput Sc 2009, 5347:70-89.

19. Benenson Y, Paz-Elizur T, Adar R, Keinan E, Livneh Z, Shapiro E: Programmable and autonomous computing machine made of biomolecules. Nature 2001, 414:430-434.

20. Benenson Y, Gil B, Ben-Dor U, Adar R, Shapiro E: An autonomous molecular computer for logical control of gene expression. Nature 2004 429:423-429.

21. Beyer S, Simmel FC: A modular DNA signal translator for the controlled release of a protein by an aptamer. Nucleic Acids Res 2006, 34:1581-1587.

22. Kim J, White KS, Winfree E: Construction of an in vitro bistable circuit from synthetic transcriptional switches. Mol Syst Biol 2006, 2:68.

23. Dirks RM, Pierce NA: Triggered amplification by hybridization chain reaction. Proc Natl Acad Sci USA 2004, 101:15275-15278.

24. Yin P, Choi HM, Calvert CR, Pierce NA: Programming biomolecular selfassembly pathways. Nature 2008, 451:318-322.

25. Travascio P, Li Y, Sen D: DNA-enhanced peroxidase activity of a DNAaptamer-hemin complex. Chem Biol 1998, 5:505-517.

26. Darius AK, Ling NJ, Mahesh U: Visual detection of DNA from salmonella and mycobacterium using split DNAzymes. Mol Biosyst 2010, 6:792-794.

27. Kolpashchikov DM: Split DNA enzyme for visual single nucleotide polymorphism typing. J Am Chem Soc 2008, 130:2934-2935.

28. Deng $M$, Zhang $D$, Zhou $Y$, Zhou $X$ : Highly effective colorimetric and visual detection of nucleic acids using an asymmetrically split peroxidase DNAzyme. J Am Chem Soc 2008, 130:13095-13102.

29. Yurke B, Turberfield AJ, Mills AP, Simmel FC, Neumann JL: A DNA-fuelled molecular machine made of DNA. Nature 2000, 406:605-608.

30. Seelig G, Soloveichik D, Zhang DY, Winfree E: Enzyme-free nucleic acid logic circuits. Science 2006, 314:1585-1588.

31. Kashida H, Takatsu T, Fujii T, Sekiguchi K, Liang X, Niwa K, Takase T, Yoshida Y, Asanuma H: In-stem molecular beacon containing a pseudo base pair of threoninol nucleotides for the removal of background emission. Angew Chem Int Ed Engl 2009, 48:7044-7047.

32. Haner R, Biner SM, Langenegger SM, Meng T, Malinovskii VL: A highly sensitive, excimer-controlled molecular beacon. Angew Chem Int Ed Engl 2010, 49:1227-1230.

33. Socher E, Jarikote DV, Knoll A, Roglin L, Burmeister J, Seitz O: FIT probes: peptide nucleic acid probes with a fluorescent base surrogate enable real-time DNA quantification and single nucleotide polymorphism discovery. Anal Biochem 2008, 375:318-330.

doi:10.1186/1759-2208-1-13

Cite this article as: Eckhoff et al:: Beyond allostery: Catalytic regulation of a deoxyribozyme through an entropy-driven DNA amplifier. Journal of Systems Chemistry 2010 1:13.

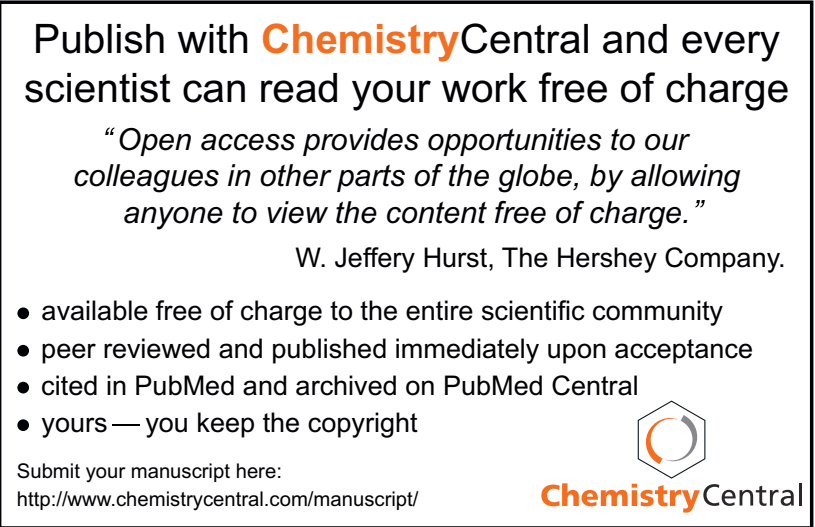

